\title{
Are self-caused distractors easier to ignore? Experiments with the flanker task
}

\author{
CiJun Gao ${ }^{1}$ • Davood G. Gozli ${ }^{1}$ \\ Accepted: 29 September 2020 / Published online: 5 November 2020 \\ (C) The Psychonomic Society, Inc. 2020
}

\begin{abstract}
Four experiments are reported that investigate the relationship between action-outcome learning and the ability to ignore distractors. Each participant performed 600 acquisition trials, followed by 200 test trials. In the acquisition phase, participants were presented with a fixed action-outcome contingency (e.g., Key \#1 $\rightarrow$ green distractors), while that contingency was reversed in the test phase. In Experiments 1-3, a distractor feature depended on the participants' action. In Experiment 1, actions determined the color of the distractors; in Experiment 2, they determined the target-distractor distance; in Experiment 3, they determined target-distractor compatibility. Results suggest that with the relatively simple features (color and distance), exposure to action-outcome contingencies changed distractor cost, whereas with the complex or relational feature (target-distractor compatibility), exposure to the contingencies did not affect distractor cost. In Experiment 4, the same pattern of results was found (effect of contingency learning on distractor cost) with perceptual sequence learning, using visual cues (" $\mathrm{X}$ " vs. " $\mathrm{O}$ ”) instead of actions. Thus, although the mechanism of associative learning may not be unique to actions, such learning plays a role in the allocation of attention to task-irrelevant events.
\end{abstract}

Keywords Associative learning $\cdot$ Ideomotor theory $\cdot$ Forward model $\cdot$ Sensory preactivation $\cdot$ Sensory attenuation $\cdot$ Flanker task $\cdot$ Visual attention

The pioneering work of Eriksen and Eriksen (1974) on visual attention introduced the flanker task, a procedure for investigating the limited capacity for controlling the spatial scope of visual attention. Studies continue to use the flanker task to study a wide range of phenomena, including perceptual grouping (Kramer \& Jacobson, 1991), cognitive control (Nieuwenhuis et al., 2006), and the effect of arousal on performance (Schneider, 2018). In the present study, we use the flanker task to examine how action-outcome learning might influence attention to task-irrelevant distractors. To do so, we compare the effect of (anticipated) self-caused distractors with (unanticipated) externally caused distractors. We ask whether the influence of taskirrelevant distractors ("flankers") can change once we

CiJun Gao

sb61152@connect.um.edu.mo

Davood G. Gozli

gozli@um.edu.mo

1 Department of Psychology, University of Macau, Taipa, Macao S.A.R., China learn the association between a distractor feature and the observer's own action.

The reason the flanker task is suited for our purpose is that it provides a setting where targets and distractors are distinguished based on their locations. Examining the effectiveness of distractors that consistently appear at irrelevant locations, helps narrow down the interpretation of findings with respect to changes in stimulus salience, rather than changes in topdown strategy. Additionally, attributes of the flanker task, including possible variations in flanker color and targetflanker distance, could be made selectively predictable.

The present study is grounded in the insight that perception is not passive or reactive, but involves actively changing the sensory input. Recent studies have emphasized the difference between passively received stimuli and stimuli that have their antecedents in the observer's own action (self-caused stimuli). These studies have demonstrated that human action, which is tied to anticipating sensory action-outcomes, influences perception (Bäß, Widmann, Roye, Schröger, \& Jacobsen, 2009; Blakemore, Wolpert, \& Frith, 2000; Bompas \& O’Regan, 2006a, 2006b; Cardoso-Leite, Mamassian, Schütz-Bosbach, \& Waszak, 2010; Gozli \& Ansorge, 2016; Hommel, Müsseler, Aschersleben, \& Prinz, 2001; Hughes \& Waszak, 
2011; Roussel, Hughes, \& Waszak, 2013, 2014; Wykowska, Schubö, \& Hommel, 2009). In explaining action-perception interface, forward models are frequently discussed. While preparing and performing an action involve generating a copy of the predicted (sensory) consequences of the motor command during action selection. The copy is referred to as an efference copy, which is a simulation or prediction of the consequence of the selected action (Miall \& Wolpert, 1996; Wolpert, Ghahramani, \& Jordan, 1995; Wolpert \& Kawato, 1998). Other general mechanisms include ideomotor theory and predictive coding (Clark, 2013; James, 1890; Lotze, 1852; Rao \& Ballard, 1999). These accounts characterize the close and necessary connection between perception and action, which in turn explains the reciprocal relation between the two.

A major phenomenon related to the action-perception interface is the sensory attenuation effect (Blakemore et al., 2000), referring to the observation that sensory events that match the prediction of self-induced actions are perceived as weaker, compared with unpredicted sensory events. This phenomenon has been described both as a neurophysiological effect and as a psychophysical effect (e.g., Aliu, Houde, \& Nagarajan, 2009; Bäß , Jacobsen, \& Schroger, 2008; Cardoso-Leite et al., 2010; Hughes \& Waszak, 2011; Schafer \& Marcus, 1973). The former describes a reduced neural response to self-caused events, whereas the latter refers to a reduced sensitivity in the subjective experience of selfcaused events. An example from everyday life is that even very ticklish people can hardly tickle themselves (Blakemore, Wolpert, \& Frith, 1998, 2000). Similarly, it has been reported that one's own speech evokes a weaker electrophysiological response compared with the speech of another person (Numminen \& Curio, 1999; Numminen, Salmelin, \& Hari, 1999; see also Dignath, Kiesel, Frings, \& Pastötter, 2019; Ford, Gray, Faustman, Heinks, \& Mathalon, 2005; Ford, Mathalon, Heinks, et al., 2001a; Ford, Mathalon, Kalba, et al., 2001b; Heinks-Maldonado, Mathalon, Gray, \& Ford, 2005; Heinks-Maldonado, Nagarajan, \& Houde, 2006; Houde, Nagarajan, Sekihara, \& Merzenich, 2002; Pfister, Heinemann, Kiesel, Thomaschke, \& Janczyk, 2012; Schmidts, Foerster, \& Kunde, 2018).

In the present study, we explore the phenomenon of sensory attenuation with the use of the flanker task, investigating whether attention is drawn less strongly toward self-caused and predictable distractors (in this case, "flankers") compared with unpredictable distractors. Our purpose is to investigate the sensory attenuation phenomenon under different circumstances, while participants learn to associate different outcomes with their own actions. The typical finding of the flanker task is better performance with target-flanker compatible trials (faster and more accurate responses), relative to targetflanker incompatible trials, which indicates attention to the irrelevant flankers. Important for our purpose, a change in the flankers' sensory salience should result in a change in the size of the flanker effect. That is, the difference between compatible and incompatible flankers should increase with more salient flankers (unanticipated), compared with less salient (anticipated, self-caused) flankers.

In each of the following Experiments 1-3, one feature of the flankers was associated with the action performed by participants. With an initial ("free-choice") response that initiated the target-flanker stimuli, participants' action determined a simple or complex feature of the flankers on that trial. By "simple" features, we refer to perceptual features that could be identified independently of the target on a particular trial. These include flanker color (Experiment 1) and target-flanker distance (Experiment 2). Of course, color and distance could be defined relative to the color or location of the target (Becker, 2013; Becker, Folk, \& Remington, 2013), but since target color and target location were both fixed throughout the experiment, flanker color and target-flanker distance could be identified by looking only at the flankers. Thus, we could treat these features as relatively simple and nonrelational.

In contrast to the relatively simple features, a complex or relational feature requires identifying both the target and the flankers on each trial. In the present study, the complex feature was target-flanker compatibility. In Experiment 3, participants' action determined target-flanker compatibility, which perhaps should not be considered as a sensory feature at all. Without sensory anticipation, there would be no sensory attenuation. As such, failing to find a learning effect despite the predictability of target-flanker compatibility would provide a boundary condition for the sensory attenuation phenomena.

Finally, in Experiment 4, we replaced participants' freechoice response with the presentation of a visual cue ("X" vs. "O"). We reasoned that participants might be able to associate the letters with the subsequent flanker color (Itti \& Baldi, 2009; Pearce \& Hall, 1980). With Experiment 4, we aimed to see whether sensory attenuation would still occur in the absence of action, given that there is some evidence against such a possibility (Cardoso-Leite et al., 2010; Logan \& Zbrodoff, 1982).

All four experiments included an acquisition phase (Blocks 1-3; 600 trials) and a test phase (Block 4; 200 trials). In each experiment, we linked only one flanker feature to the participants' actions, while other features varied randomly. The action-outcome contingency was constant in the acquisition phase, and it was reversed in the test phase. In line with previous observations, we predicted that participants would learn the particular contingency, which would be reflected in (1) a reduced flanker effect across Blocks 1-3 (sensory attenuation) and (2) an increased flanker effect in Block 4, when the actionoutcome contingencies are reversed, due to violating the acquired expectations (sensory amplification).

Given the use of free-choice responses (which were associated with a distractor feature), we should consider the possible role of choice probabilities. In particular, in a setup 
where the free-choice response determines the color of flankers (Experiment 1), favoring of one response over the alternative (e.g., 60\%) might produce a general predictability of the corresponding flanker feature (e.g., flankers are $60 \%$ likely to be green), which is then reversed in the test phase. In Experiment 3, where the free-choice response determines target-flanker compatibility, some participants might favor the response that results in target-flanker compatibility, over incompatibility. If so, then target-flanker compatible trials become more frequent than incompatible trials in the acquisition phase, which is then reversed in the test phase. This leads to an alternative interpretation based on relatively long-term predictability of events, which differs from our interpretation based on learning specific response-outcome associations. Unfortunately, we could not include choice probabilities in the current analyses because we did not have access to the necessary software. ${ }^{1}$ But given the availability of the data on Open Science Framework (https://osf.io/x28hy), follow-up analyses of choice probabilities and their impact is possible.

\section{General method}

\section{Participants}

We recruited a total of 157 University of Macau undergraduate students for the experiments in exchange for course credit ( 0.5 per experiment) or coupons usable at a local café. Based on the effect sizes observed in previous studies (Cardoso-Leite et al., 2010; Gozli \& Ansorge, 2016; Gozli, Aslam, \& Pratt, 2016a; Gozli, Huffman, \& Pratt, 2016b), we estimated that recruiting 20 or more participants in each experiment would be sufficient ( $\alpha=.05,1-\beta=.8$ ), for detecting a simple contrast between the condition that confirms a learned association (e.g., flanker effect in Block 3 in the present study) and the condition that violates the learned association (e.g., flanker effect in Block 4 in the present study). Previous studies with comparable procedures had recruited 16-26 participants per experiment (CardosoLeite et al., 2010; Gozli \& Ansorge, 2016; Gozli et al., 2016a, b). Compared with previous studies, we extended our acquisition phase, and consequently, we expected our experiments to offer a robust test of the hypotheses. In addition, we decided to recruit at least 36 participants per experiment. We did not dismiss any participants who had already registered for an experiment, so we continued admitting participants who had signed up, even after reaching our minimum requirement. Moreover, we had to later exclude five participants from analysis due to their failure to follow

\footnotetext{
${ }^{1}$ This is due to a COVID-9 travel restriction, which has prevented the authors' access to lab/office computers, which contain the necessary software for additional analyses.
}

task instructions (i.e., either selecting one of the "freechoice" responses too frequently or following an obvious pattern of responding; see Naefgen \& Janczyk, 2018). The final analyses included 36, 34, 44, and 38 participants, respectively, for Experiments 1-4. All participants reported normal or corrected-to-normal vision and were unaware of the purpose of the study. All experimental protocols were approved by the local ethics committee at the Faculty of Social Sciences of the University of Macau. All data are available on the Open Science Framework (https://osf.io/ x28hy).

\section{Apparatus and stimuli}

Participants performed the task in a well-lit room. Stimuli were presented on a 21-in LCD monitor, set at a 1,920 $\times$ 1,080 resolution and a refresh rate of $60 \mathrm{~Hz}$. Participants sat at the viewing distance of about $55-75 \mathrm{~cm}$ from the monitor, and we did not control their viewing distance with a head/chin rest. For this reason, we have described the properties of the stimuli in centimeters rather than degrees of visual angle. The display structure and the sequence of events in a sample trial are shown in Fig. 1. A trial began with the presentation of a fixation cross (RGB: 250, 250, 250; $0.8 \times 0.8 \mathrm{~cm}$ ). Targets and flankers consisted of arrowheads (">" and "<") that could each fit in a $0.5 \times 0.5 \mathrm{~cm}$ rectangle. On each trial, a target and two flankers were presented together, fitting in a $3.0 \times 0.5$ (for "near" flankers) or $5.5 \times 0.5 \mathrm{~cm}$ ("far" flankers) rectangle. The target's color appeared dark green (RGB: 50, 50, 10). The flankers' colors were red (RGB: 255, 0, 0) or green (RGB: 0, 130,0). All stimuli were presented against a black background (RGB: 0,0,0). The target was consistently presented at the display center, and flankers appeared to the left and right of the target. The two flankers on a given trial always had the same color.

Participants performed two responses per trial. First, was the "free-choice" response that initiated the onset of the targetflanker stimuli and was linked to a particular visual effect. This initial response was performed using the index and the middle fingers of the left hand with the " $\mathrm{A}$ " and "Q" buttons on a QWERTY keyboard. We associated left-hand responses with action outcomes based on prior research suggesting that action-outcome learning may be stronger with the nondominant hand (Melcher, Weidema, Eenshuistra, Hommel, \& Gruber, 2008; Melcher et al., 2013). The second response was performed with the right hand, using the left and right arrow keys (in response to the "<" and ">" targets, respectively).

Each experiment consisted of 10 practice trials and 800 experimental trials. Experimental trials were divided into four blocks (200 trials each). The first three blocks (600 trials) comprised the "acquisition" phase, and the final $200 \mathrm{com}$ prised the "test" phase. After every 100 trials, participants had an opportunity to take a self-paced break. 


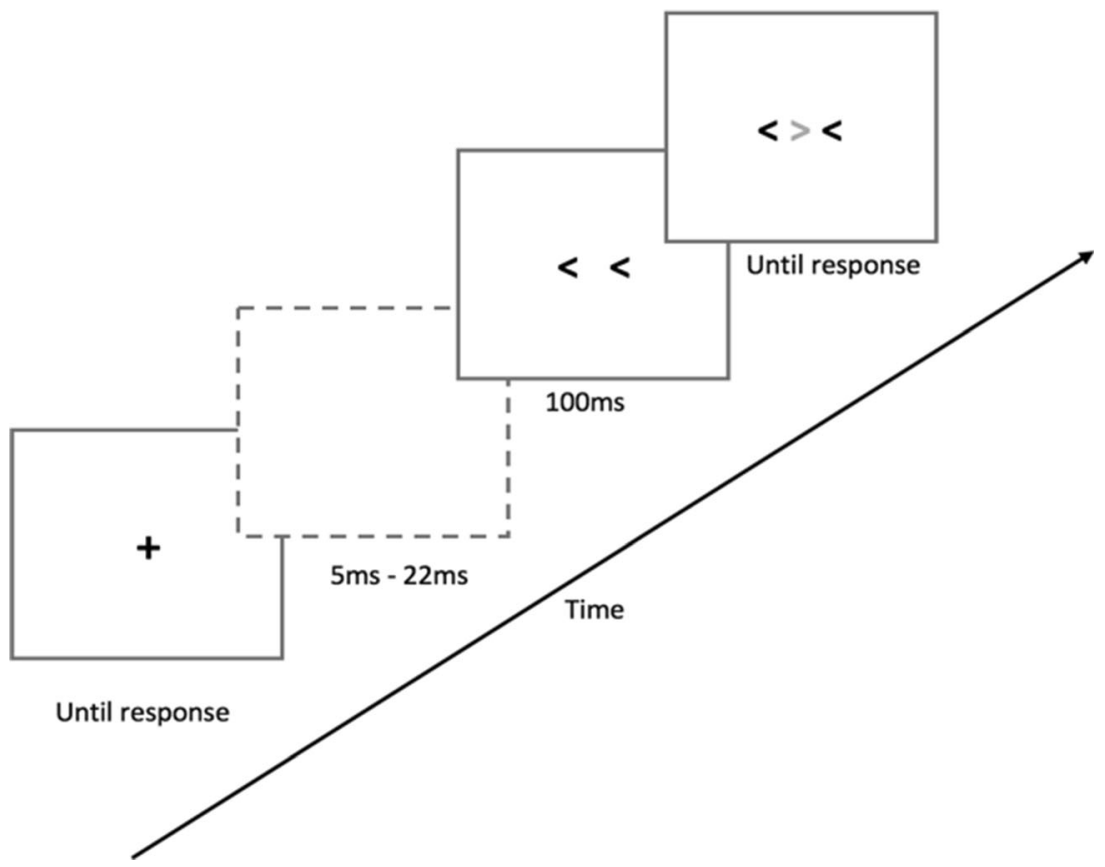

Fig. 1 Sequence of events on a sample trial of the acquisition phase

\section{Procedure: I. Acquisition phase}

Each trial of the acquisition phase began with the presentation of a fixation cross at the display center. We instructed participants to press either the " $Q$ " or the " $A$ " upon noticing the cross. We asked participants to choose their left-hand responses spontaneously and try to avoid predictable patterns (e.g., "AQAQA ..."; Naefgen \& Janczyk, 2018). There is evidence suggesting that action-outcome associations might be acquired and/or recruited more robustly with this type of response (Gozli et al., 2016b; Pfister, Kiesel, \& Hoffmann, 2011; Pfister, Kiesel, \& Melcher 2010; see also, Gozli, 2019; Huffman, Gozli, Hommel, \& Pratt, 2019; Wolfensteller \& Ruge, 2014). If participants pressed a key other than " $\mathrm{Q}$ " or "A," or if they pressed more than one key, they would receive visual feedback ("MISTAKE") and no target-flanker display followed. Immediately after a correct first response was recorded ("Q" or " $A$ "), the flankers appeared at the left and right of the center. The target appeared after a 100-ms delay following the presentation of the flankers. We introduced the 100-ms flanker-target onset asynchrony to increase the chance of the flankers being attended and influencing performance. We reasoned that with a robust baseline flanker effect we would more easily obtain modulations of the flanker effect.

For the second response during the trial (i.e., the targetdirected response), we instructed participants to identify the target orientation (">" = right, " $<"=$ left) using the left/right arrow keys on the keyboard. In all the following experiments, trials differed with respect to target-flanker compatibility, flanker color (red vs. green), and target-flanker distance (near vs. far). The flankers and target remained on the screen until a response was recorded. Upon pressing an incorrect key, or more than one key, participants received a visual feedback ("MISTAKE"). The next trial began after the response for orientation was recorded or a visual feedback was presented.

Experiments 1-3 differed with respect to the outcome of the first (left-hand) response. Depending on the experiments, the left-hand response determined the flanker color (Experiment 1), target-flanker distance (Experiment 2), or the targetflanker compatibility (Experiment 3). The final Experiment 4, which served as a control experiment to Experiment 1, the first (left-hand) response was removed, and flanker colors were associated with visual cues ("X" and "O"). Participants were not informed at the beginning about the action-outcome or stimulus-outcome contingencies. During informal debriefing conversations with the participants, we found that they were either unaware of the contingencies or had a vague idea about it, which they did not further explore while performing the task.

\section{Procedure: II. Test (reversal) phase}

In the test phase, the action-outcome contingencies were reversed. For example, if the action determined the flanker color ("Q" $\rightarrow$ red, "A" $\rightarrow$ green), the mapping between the response and the color were reversed in the test phase ("Q" $\rightarrow$ green, "A" $\rightarrow$ red). The stimuli and the order of events were otherwise the same as the acquisition phase. Participants were not told that they were entering a different phase of the experiment. Indeed, we found during debriefing conversations that the participants were either unaware of the change occurring in the test (reversal) phase or had a vague idea, to which they did not pay much attention. 


\section{Experiment 1}

\section{Method}

Thirty-six participants performed in the experiment (26 females; mean age $=21$ years, $S D=2.71$ years). In Experiment 1, the first response of each trial determined flanker colors (during the acquisition phase: red after " $\mathrm{Q}$ " and green after "A"). Action-outcome associations were reversed in the test phase. Target-flanker distance (near vs. far) and targetflanker compatibility (compatible vs. incompatible) were pseudorandomized and equiprobable.

\section{Results}

Response time RT data were submitted to a $4 \times 2 \times 2$ repeatedmeasures analysis of variance (ANOVA), with the factors block, target-flanker distance, and target-flanker compatibility (see Table 1). The analysis revealed main effects of block, distance, and compatibility. The main effect of block indicates faster responses on the final block ( $M \pm S E$ for Blocks 1-4, respectively, $463 \pm 13,461 \pm 12,460 \pm 10$, and $448 \pm 11 \mathrm{~ms}$ ), showing a benefit of practice. The main effect of distance indicates faster responses with far flankers $(M \pm S E=456 \pm$ $11 \mathrm{~ms})$ than with near flankers $(460 \pm 11 \mathrm{~ms})$. The main effect of compatibility shows faster responses on target-flanker compatible trials $(415 \pm 12 \mathrm{~ms})$ compared with incompatible trials $(502 \pm 11 \mathrm{~ms})$

Table 1. Results of ANOVAs on response time (RT) and percentage error (PE) in Experiment 1

\begin{tabular}{llllll}
\hline Factor & $d f$ & $M S E$ & $F$ & $p$ & $\eta_{\mathrm{p}}{ }^{2}$ \\
\hline & $R T$ & & & & \\
Block (B) & $\mathbf{3}$ & $\mathbf{6 , 3 3 2 . 0 7}$ & $\mathbf{4 . 7 3}$ & $<.001$ & $\mathbf{0 . 1 4}$ \\
Distance (D) & $\mathbf{1}$ & $\mathbf{1 , 9 1 3 . 1 2}$ & $\mathbf{1 5 . 0 5}$ & $<.001$ & $\mathbf{0 . 3 3}$ \\
Compatibility (C) & $\mathbf{1}$ & $\mathbf{9 4 3 , 8 9 1 . 4 4}$ & $\mathbf{4 4 8 . 1 8}$ & $<.001$ & $\mathbf{0 . 9 4}$ \\
B $\times$ D & 3 & 312.15 & 2.38 & .08 & 0.07 \\
B $\times$ C & $\mathbf{3}$ & $\mathbf{1 , 7 1 8 . 6 1}$ & $\mathbf{5 . 5 5}$ & $<.001$ & $\mathbf{0 . 1 6}$ \\
D $\times$ C & $\mathbf{1}$ & $\mathbf{2 0 , 7 7 4 . 9 1}$ & $\mathbf{1 0 4 . 3 2}$ & $<.001$ & $\mathbf{0 . 7 8}$ \\
B $\times$ D $\times$ C & $\mathbf{3}$ & $\mathbf{6 3 6 . 5 3}$ & $\mathbf{4 . 1 1}$ & $\mathbf{. 0 1}$ & $\mathbf{0 . 1 2}$ \\
& $P E$ & & & & \\
Block(B) & $\mathbf{3}$ & $\mathbf{0 . 0 1 6 8 4 9}$ & $\mathbf{7 . 7 8}$ & $<.001$ & $\mathbf{0 . 2 1}$ \\
Distance (D) & $\mathbf{1}$ & $\mathbf{0 . 0 3 6 4 2 5}$ & $\mathbf{2 2 . 8 3}$ & $<.001$ & $\mathbf{0 . 4 3}$ \\
Compatibility(C) & $\mathbf{1}$ & $\mathbf{0 . 4 0 7 2 5 2}$ & $\mathbf{3 4 . 1 7}$ & $<.001$ & $\mathbf{0 . 5 3}$ \\
B $\times$ D & $\mathbf{3}$ & $\mathbf{0 . 0 0 2 8 3 2}$ & $\mathbf{5 . 1 1}$ & $<.001$ & $\mathbf{0 . 1 5}$ \\
B $\times$ C & $\mathbf{3}$ & $\mathbf{0 . 0 1 3 3 2 3}$ & $\mathbf{8 . 7 4}$ & $<.001$ & $\mathbf{0 . 2 3}$ \\
D $\times$ C & $\mathbf{1}$ & $\mathbf{0 . 0 3 9 1 6 3}$ & $\mathbf{3 1 . 6 4}$ & $<.001$ & $\mathbf{0 . 5 1}$ \\
B $\times$ D $\times$ C & $\mathbf{3}$ & $\mathbf{0 . 0 0 3 1 9 0}$ & $\mathbf{5 . 1 0}$ & $<.001$ & $\mathbf{0 . 1 5}$ \\
\hline
\end{tabular}

Note. Boldface indicates a significant effect at alpha $=0.05$
In addition to the main effects, we found an interaction between distance and compatibility, which shows a larger flanker effect with near flankers (flanker effect $=100 \pm 4$ $\left.\mathrm{ms}, d_{z}=4.14\right)$ compared with far flankers (74 $\pm 4 \mathrm{~ms}, d_{z}=$ 3.12). Most importantly for our purpose, we found a Block $\times$ Compatibility interaction (see Fig. 2). This interaction shows that the flanker effect decreased from the first block to the third block, which might have partly been due to acquiring the consistent action-outcome contingencies (sensory attenuation of predicted colors). The flanker effect then increased on the final block, which could suggest the cost of violating the previously learned action-outcome contingencies (sensory amplification of unpredicted colors). We should note that the increased flanker effect in Block 4 happened despite the general practice effect on improving performance. These results are consistent with the effect of action-outcome learning on attention to the flankers.

Moreover, we found a three-way interaction, which can be described with reference to our hypothesis. The increase in the flanker effect from Block 3 to Block 4 was slightly more pronounced with far flankers (flanker effect changing from 67 to $77 \mathrm{~ms}$ ) than with near flankers (from 90 to $98 \mathrm{~ms}$ ). This might mean that violating predictions about action-
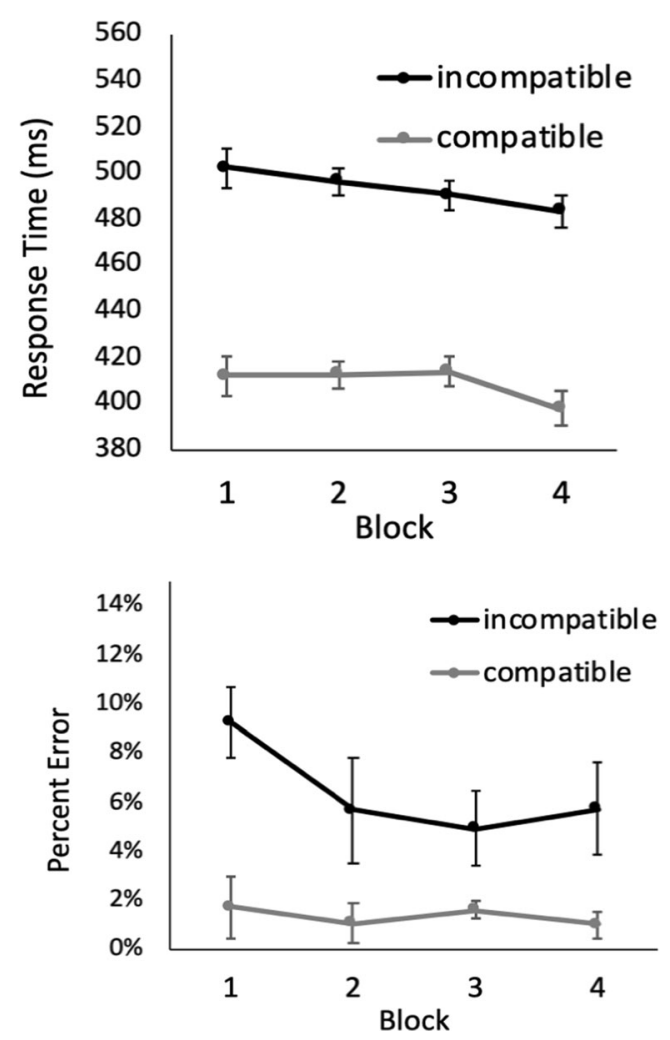

Fig. 2 Response time (RT) and percentage error (PE) data from Experiment 1, graphed as a function of target-flanker compatibility (compatible vs. incompatible), block (Blocks 1-3: acquisition of a consistent action-outcome association; Block 4: reversal of the acquired association). Error bars represent $95 \%$ within-subjects confidence intervals (Cousineau, 2005; Morey, 2008) 
outcome contingencies had a weaker effect when the flankers were physically more salient (near flankers) than when they were physically less salient (far flankers).

Percentage error PE data were submitted to the same repeated-measures ANOVA (see Table 1), which revealed main effects of block, distance, and compatibility. The main effect of block indicates a trend of decreasing errors over Blocks 1-3 (mean PEs, respectively, 5.27\%, 3.04\%, and $2.70 \%$,), and an increase in PE on Block $4(M=3.21 \%)$. The main effect of distance shows smaller PE with far flankers $(M=2.70 \%)$ than with near flankers $(M=4.41 \%)$. The main effect of compatibility indicates smaller PE on target-flanker compatible trials $(M=0.69 \%)$ than incompatible trials $(M=$ $6.42 \%)$.

Additionally, we found a Block $\times$ Distance interaction, which shows an increase in the effect of distance over Blocks 1-4 (respectively, 0.36\%, 2.09\%, 1.81\%, and $2.59 \%$ ), and a Distance $\times$ Compatibility interaction, due to a large flanker effect with near flankers (flanker effect $=7.51 \%$, $\left.d_{z}=1.12\right)$ compared with far flankers $\left(3.95 \%, d_{z}=0.86\right)$. Most important for our purpose, we found an interaction between block and compatibility (see Fig. 2), which shows a decrease in flanker effect across Blocks 1-3 (respectively, 8.52\%, $5.36 \%$, and $3.51 \%$ ) and an increase in the flanker effect on Block $4(M=5.53 \%)$. These are consistent with the RT results.

We also found a three-way interaction, which we could describe with reference to our hypothesis. The increase in the flanker effect from Block 3 to Block 4 was more pronounced with near flankers (from $5.55 \%$ to $8.01 \%$ ) than with far flankers (from $1.47 \%$ to $3.05 \%$ ). This might mean that the violating predictions about action-outcome contingencies had a stronger effect when the flankers were physically more salient (near flankers) than when they were physically less salient (far flankers).

\section{Experiment 2}

\section{Method}

Thirty-six participants performed in this experiment ( $26 \mathrm{fe}-$ males; mean age $=19$ years, $S D=1.22$ ). Two participants were excluded due to the failure to follow task instructions. In Experiment 2, the first response of the trial determined target-flanker distance (during the acquisition phase: near flankers after "Q" and far flankers after "A"). Actionoutcome associations were then reversed in the test phase. Target-flanker color (red vs. green) and target-flanker compatibility were pseudorandomized and equiprobable.

\section{Results}

Response time RT data were submitted to a $4 \times 2 \times 2$ repeatedmeasures ANOVA, with factors block, target-flanker distance, and target-flanker compatibility (see Table 2).

The analysis revealed main effects of block, distance, and compatibility. The main effect of block indicates a benefit of practice, with relatively faster responses on Block $4(M \pm S E$ for Blocks 1-4, respectively, $444 \pm 7,445 \pm 6,437 \pm 5$, and $425 \pm 5 \mathrm{~ms})$. The main effect of distance indicates faster responses with far flankers $(M \pm S E=436 \pm 5 \mathrm{~ms})$ than with near flankers ( $440 \pm 5 \mathrm{~ms})$. The main effect of compatibility shows faster responses on target-flanker compatible trials (398 \pm 6 $\mathrm{ms}$ ) than incompatible trials $(478 \pm 5 \mathrm{~ms})$.

In addition to the main effects, we found a Distance $\times$ Compatibility interaction, due to a larger flanker effect with near flankers (flanker effect $=90 \pm 26 \mathrm{~ms}, d_{z}=3.46$ ) compared with far flankers ( $\left.69 \pm 22 \mathrm{~ms}, d_{z}=3.17\right)$. Most important for our purpose, we found an interaction between block and compatibility (see Fig. 3). From Block 1 to Block 3, the flanker effect showed a decreasing trend, which could suggest the benefit of consistent action-outcome contingencies. On the final block, however, the flanker effect increased, presumably showing a cost of violating the learned action-outcome contingencies.

Percentage error The error data were submitted to the same repeated-measures ANOVA (see Table 2), which revealed main effects of block, distance, and compatibility. The main

Table 2. Results of ANOVAs on response time (RT) and percentage error (PE) in Experiment 2

\begin{tabular}{llllll}
\hline Factor & $d f$ & $M S E$ & $F$ & $p$ & $\eta_{\mathrm{p}}{ }^{2}$ \\
\hline & $R T$ & & & & \\
Block (B) & $\mathbf{3}$ & $\mathbf{1 2 , 2 4 1 . 4 2}$ & $\mathbf{1 2 . 7 6}$ & $<.001$ & $\mathbf{0 . 2 7 9}$ \\
Distance (D) & $\mathbf{1}$ & $\mathbf{1 , 9 3 9 . 6 0}$ & $\mathbf{1 3 . 8 0}$ & $\mathbf{. 0 0 1}$ & $\mathbf{0 . 2 9 5}$ \\
Compatibility (C) & $\mathbf{1}$ & $\mathbf{8 6 2 , 2 1 4 . 5 4}$ & $\mathbf{4 1 2 . 4 9}$ & $<.001$ & $\mathbf{0 . 9 2 6}$ \\
B $\times$ D & 3 & 88.11 & 0.69 & .563 & 0.020 \\
B $\times$ C & $\mathbf{3}$ & $\mathbf{1 , 7 9 9 . 2 8}$ & $\mathbf{7 . 4 6}$ & $<.001$ & $\mathbf{0 . 1 8 4}$ \\
D $\times$ C & $\mathbf{1}$ & $\mathbf{1 4 , 6 7 8 . 1 6}$ & $\mathbf{6 6 . 4 0}$ & $<.001$ & $\mathbf{0 . 6 6 8}$ \\
B $\times$ D $\times$ C & 3 & 60.31 & 0.44 & .728 & 0.013 \\
& $P E$ & & & & \\
Block (B) & $\mathbf{3}$ & $\mathbf{0 . 0 1}$ & $\mathbf{4 . 4 8}$ & $\mathbf{. 0 0 5}$ & $\mathbf{0 . 1 2 0}$ \\
Distance (D) & $\mathbf{1}$ & $\mathbf{0 . 0 4}$ & $\mathbf{2 3 . 2 3}$ & $<.001$ & $\mathbf{0 . 4 1 3}$ \\
Compatibility (C) & $\mathbf{1}$ & $\mathbf{0 . 3 0}$ & $\mathbf{4 2 . 7 5}$ & $<.001$ & $\mathbf{0 . 5 6 4}$ \\
B $\times$ D & $\mathbf{3}$ & $\mathbf{0 . 0 1}$ & $\mathbf{1 0 . 8 3}$ & $<.001$ & $\mathbf{0 . 2 4 7}$ \\
B $\times$ C & $\mathbf{3}$ & $\mathbf{0 . 0 1}$ & $\mathbf{1 4 . 6 2}$ & $<.001$ & $\mathbf{0 . 3 0 7}$ \\
D $\times$ C & $\mathbf{1}$ & $\mathbf{0 . 0 4}$ & $\mathbf{2 7 . 1 1}$ & $<.001$ & $\mathbf{0 . 4 5 1}$ \\
B $\times$ D $\times$ C & $\mathbf{3}$ & $\mathbf{0 . 0 0}$ & $\mathbf{4 . 7 4}$ & $\mathbf{. 0 0 4}$ & $\mathbf{0 . 1 2 6}$ \\
\hline
\end{tabular}

Note. Boldface indicates a significant effect at alpha $=0.05$ 

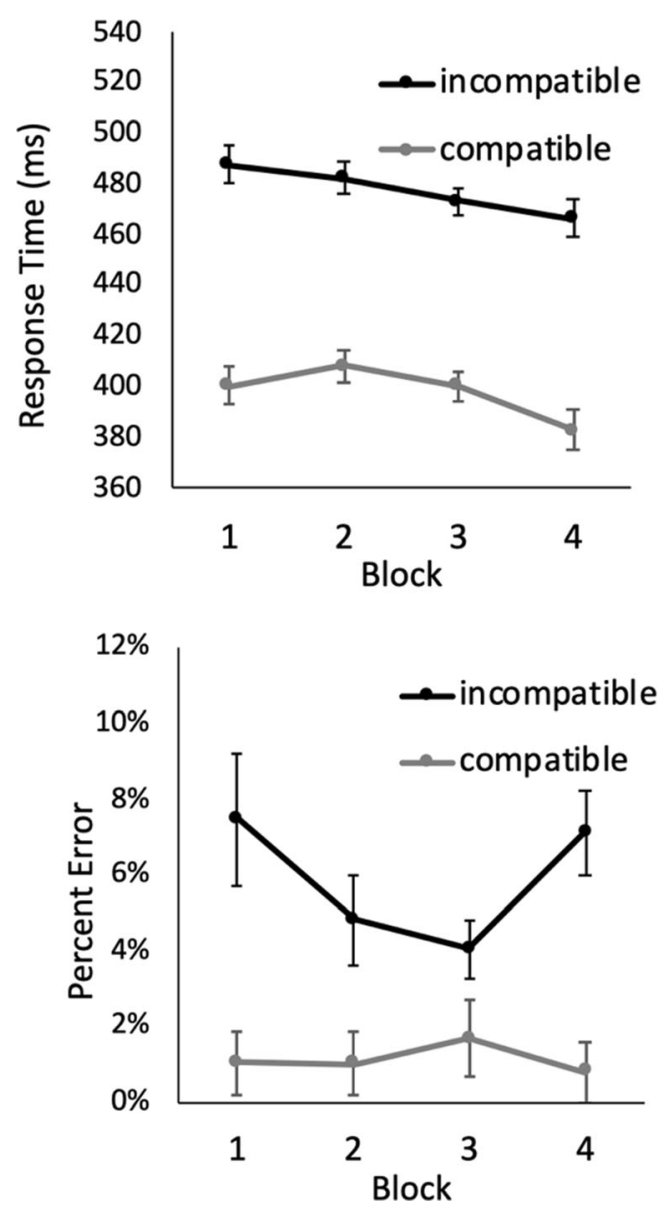

Fig. 3 Response time (RT) and percentage error (PE) data from Experiment 2, graphed as a function of target-flanker compatibility (compatible vs. incompatible), block (Blocks 1-3: acquisition of a consistent action-outcome association; Block 4: reversal of the association). Error bars represent $95 \%$ within-subjects confidence intervals (Cousineau, 2005; Morey, 2008)

effect of block indicates a trend of decreasing errors over Blocks 1-3 (respectively, $4.25 \%, 2.90 \%$, and $2.85 \%$ ), and an increase in errors on Block $4(M=3.94 \%)$. The main effect of distance shows smaller PE with far flankers (2.67\%) than with near flankers $(4.31 \%)$. The main effect of compatibility indicates smaller PE on target-flanker compatible trials $(1.12 \%)$ than on incompatible trials $(5.85 \%)$.

Additionally, we found a Block $\times$ Distance interaction, which indicates an increase in the effect of distance over Blocks 1-4 (respectively, $0.01 \%, 1.09 \%, 2.30 \%$, and $3.14 \%$ ), and a Distance $\times$ Compatibility interaction, due to a larger flanker effect with near flankers (flanker effect $=6.53 \%$, $\left.d_{z}=1.19\right)$ compared with far flankers $\left(2.94 \%, d_{z}=0.84\right)$. Most important for our purpose, we found an interaction between block and compatibility (see Fig. 3), which shows reduction of flanker effect across Blocks 1-3 (respectively, $6.43 \%, 3.81 \%$, and $2.36 \%$ ) and an increase in the flanker effect on Block 4 $(6.34 \%)$.

We also found a three-way interaction, which we could describe in terms of our hypothesis. The increase in the flanker effect from Block 3 to 4 was more pronounced with near flankers (from $3.3 \%$ to $9.6 \%$ ) than with far flankers (from $1.4 \%$ to $3.1 \%$ ). This could mean that violating the previous associations between actions and outcomes had a stronger effect when the flankers were physically more salient (near flankers) than when they were physically less salient (far flankers).

\section{Experiment 3}

\section{Method}

Forty-six participants performed in this experiment (33 females; mean age $=19$ years, $S D=1.18$ years). Two participants were excluded due to the failure to follow task instructions. The first response of the trial determined target-flanker compatibility (during acquisition, "Q" $\rightarrow$ compatible, "A" $\rightarrow$ incompatible). Action-outcome contingencies were reversed in Block 4. Flanker color (red vs. green) and target-flanker distance (near vs. far) were pseudorandomized and equiprobable.

\section{Results}

Response time RT data were submitted to a $4 \times 2 \times 2$ repeatedmeasures ANOVA, with factors block, target-flanker distance, and target-flanker compatibility (see Table 3). The analysis revealed main effects of block, distance, and

Table 3. Results of ANOVAs on response time (RT) and percentage error (PE) in Experiment 3

\begin{tabular}{llllll}
\hline Factor & $d f$ & $M S E$ & $F$ & $p$ & $\eta_{\mathrm{p}}{ }^{2}$ \\
\hline & $R T$ & & & & \\
Block (B) & $\mathbf{3}$ & $\mathbf{6 , 2 7 3 . 6 5}$ & $\mathbf{4 . 2 5}$ & $\mathbf{. 0 0 7}$ & $\mathbf{0 . 0 9}$ \\
Distance (D) & $\mathbf{1}$ & $\mathbf{4 , 3 2 5 . 3 2}$ & $\mathbf{2 7 . 2 5}$ & $<.001$ & $\mathbf{0 . 3 8 8}$ \\
Compatibility (C) & $\mathbf{1}$ & $\mathbf{1 , 4 4 4 , 1 0 9 . 6 9}$ & $\mathbf{5 6 4 . 8 8}$ & $<.001$ & $\mathbf{0 . 9 2 9}$ \\
B $\times$ D & 3 & 305.89 & 2.65 & .052 & 0.058 \\
B $\times$ C & 3 & 761.81 & 1.98 & .121 & 0.044 \\
D $\times$ C & $\mathbf{1}$ & $\mathbf{3 0 , 0 1 7 . 6 3}$ & $\mathbf{1 8 3 . 5 1}$ & $<.001$ & $\mathbf{0 . 8 1}$ \\
B $\times$ D $\times$ C & 3 & 140.95 & 140.95 & .352 & 0.025 \\
& $P E$ & & & & \\
Block (B) & 3 & 0.000616 & 0.32 & .81 & 0.01 \\
Distance (D) & $\mathbf{1}$ & $\mathbf{0 . 0 3 8 7 0 5}$ & $\mathbf{6 1 . 3 7}$ & $<.001$ & $\mathbf{0 . 5 9}$ \\
Compatibility (C) & $\mathbf{1}$ & $\mathbf{0 . 4 3 6 0 0 9}$ & $\mathbf{7 3 . 9 7}$ & $<.001$ & $\mathbf{0 . 6 3}$ \\
B $\times$ D & 3 & 0.000172 & 0.22 & .88 & 0.01 \\
B $\times$ C & 3 & 0.001036 & 0.63 & .60 & 0.01 \\
D $\times$ C & $\mathbf{1}$ & $\mathbf{0 . 0 3 9 9 0 1}$ & $\mathbf{4 0 . 3 7}$ & $<.001$ & $\mathbf{0 . 4 8}$ \\
B $\times$ D $\times$ C & 3 & 0.000440 & 0.69 & .56 & 0.02 \\
\hline
\end{tabular}

Note. Boldface indicates a significant effect at alpha $=0.05$ 
compatibility. The main effect of block indicates increasingly faster responses with performance ( $M \pm S E$ for Blocks 1-4, respectively, $457 \pm 7,456 \pm 8,456 \pm 6$, and $445 \pm 5 \mathrm{~ms}$ ). The main effect of distance indicates faster responses with far flankers $(M \pm S E=451 \pm 6 \mathrm{~ms})$ than with near flankers (456 $\pm 6 \mathrm{~ms}$ ). The main effect of compatibility indicates faster responses on target-flanker compatible trials (408 $\pm 6 \mathrm{~ms})$ than incompatible trials (499 $\pm 7 \mathrm{~ms})$.

In addition to the main effects, we found an interaction between distance and compatibility, due to a larger flanker effect with near flankers (flanker effect $=104 \pm 4 \mathrm{~ms}, d_{z}=$ 3.73 ) compared with far flankers ( $\left.78 \pm 4 \mathrm{~ms}, d_{z}=3.20\right)$. Most important for our purpose, we found no interaction between block and compatibility (see Fig. 4). In fact, the trend shown in Fig. 3 suggests a reduction in the flanker effect on Block 4 , inconsistent with action-outcome learning.
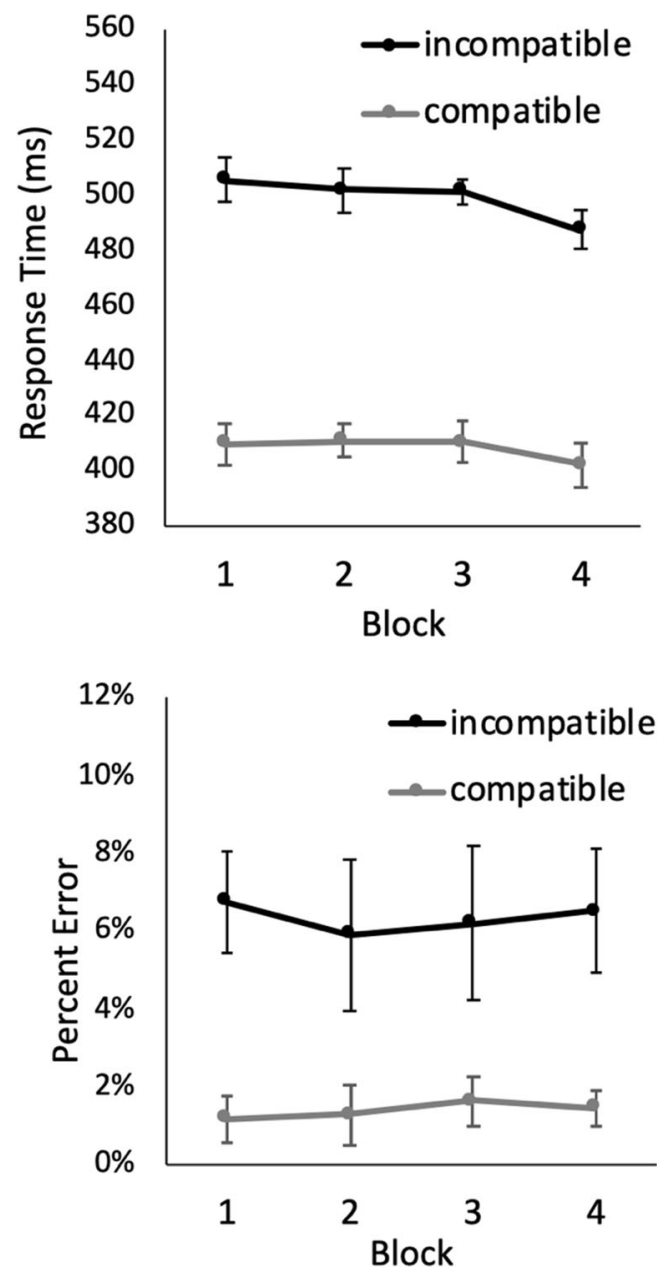

Fig. 4 Response time (RT) and percentage error (PE) data from Experiment 3, graphed as a function of target-flanker compatibility (compatible vs. incompatible), block (Blocks 1-3: acquisition of a consistent action-outcome association; Block 4: reversal of the acquired association). Error bars represent $95 \%$ within-subjects confidence intervals (Cousineau, 2005; Morey, 2008)
Percentage errors PE data were submitted to the same $4 \times 2 \times$ 2 repeated-measures ANOVA (see Table 3). The analysis revealed main effects of distance and compatibility. The main effect of distance shows larger PE with near flankers $(M=$ $4.61 \%)$ than far flankers $(M=3.12 \%)$. The main effect of compatibility shows smaller PE on target-flanker compatible trials $(M=1.38 \%)$ than on incompatible trials $(M=6.35 \%)$. Additionally, we found an interaction between distance and compatibility, which indicates a larger flanker effect (flanker effect $=6.46 \%, d_{z}=1.33$ ) on near flanker trials than on far flanker trials $\left(3.48 \%, d_{z}=1.06\right)$. Matching the RT results, we did not find an interaction between block and compatibility (see Fig. 4).

\section{Experiment 4}

The aim of this experiment was to test whether action is unique in serving as a source of perceptual prediction, at least in our flanker task, or whether action-outcome associative learning represents one possible way for perceptual prediction (e.g., Clark, 2013; Press, Kok, \& Yon, 2019, 2020). We replaced the first (left-hand) response with a perceptual cue (" $\mathrm{X}$ " vs. "O"). Otherwise, Experiment 4 was similar to Experiment 1 , particularly since the flanker color was the predictable feature. Although not providing any instructions regarding the visual cues might render them ineffective (Logan \& Zbrodoff, 1982), this is consistent with how free-choice responses were introduced to the participants in the other experiments (i.e., without drawing attention to their predictive value).

\section{Method}

Thirty-nine participants performed in this experiment (17 females, mean age $=19$ years, $S D=0.99$ years). One participant was excluded due to the failure to follow task instructions. Participants perform only one response per trial. The experiment started with a gray-green visual cue ("X" or "O") presented at the display center for 1,000 ms. Upon the automatic offset of the cue, colored flankers appeared followed by, after a 100-ms delay, the target onset. Similar to Experiments 1-3, participants were instructed to respond to the target with left/ right arrow key. Visual cues ("X"/ "O") were pseudorandomized and equiprobable. The cue predicted flanker color on each trial with $100 \%$ reliability (during the acquisition phase: "X" $\rightarrow$ red flankers, "O" $\rightarrow$ green flankers). The cue-color associations were reversed in the test phase (i.e., Block 4). Target-flanker distance (near vs. far) and target-flanker compatibility were pseudorandomized and equiprobable. 


\section{Results}

Response time RT data were submitted to a $4 \times 2 \times 2$ repeatedmeasure ANOVA, with factors block, target-flanker distance, and target-flanker compatibility (see Table 4). The analysis revealed main effects of block and compatibility. The main effect of block indicates relatively faster responses on Block 4 ( $M \pm S E$ for Blocks 1-4, respectively, $486 \pm 10,488 \pm 11,478$ \pm 9 , and $465 \pm 7 \mathrm{~ms}$ ). The main effect of compatibility shows faster responses with target-flanker compatible trials (442 \pm 8 $\mathrm{ms})$ than target-flanker incompatible trials $(517 \pm 8 \mathrm{~ms})$.

Besides the main effects, we found an interaction between distance and compatibility, with larger flanker effect with near flankers (flanker effect $=85 \pm 4 \mathrm{~ms}, d_{z}=3.20$ ) compared with far flankers $\left(65 \pm 4 \mathrm{~ms}, d_{z}=2.95\right)$. Consistent with our hypothesis, we found Block $\times$ Compatibility interaction (see Fig. 5), which shows decreasing flanker effect over Blocks 1-3 (flanker effect for Blocks 1-3, respectively, $87 \pm 5,74 \pm 6$, and $66 \pm 6 \mathrm{~ms}$ ), and an increase in the flanker effect on Block 4 (74 $\pm 6 \mathrm{~ms})$.

Percentage error PE data were submitted to the same repeated-measure ANOVA (see Table 4) that revealed main effects of distance and compatibility. The main effect of distance shows larger PE with near flankers $(M=2.5 \%)$ compared with far flankers $(M=2.1 \%)$. The main effect of compatibility indicates larger $\mathrm{PE}$ with target-flanker incompatible trials $(M=3.8 \%)$ than target-flanker compatible trials $(M=$ $0.8 \%$ ). In addition to the main effects, we found a Block $\times$

Table 4. Results of ANOVAs on response time (RT) and percentage error (PE) in Experiment 4

\begin{tabular}{llllll}
\hline Factor & $d f$ & $M S E$ & $F$ & $p$ & $\eta_{\mathrm{p}}{ }^{2}$ \\
\hline & $R T$ & & & & \\
Block (B) & $\mathbf{3}$ & $\mathbf{1 6 , 5 5 9 . 0 0}$ & $\mathbf{4 . 5 9}$ & $\mathbf{. 0 1}$ & $\mathbf{0 . 1 1}$ \\
Distance (D) & 1 & $1,238.53$ & 2.26 & .14 & 0.06 \\
Compatibility (C) & $\mathbf{1}$ & $\mathbf{8 6 0 , 6 8 6 . 1 7}$ & $\mathbf{3 9 2 . 0 3}$ & $<.001$ & $\mathbf{0 . 9 1}$ \\
B $\times$ D & 3 & 44.44 & 0.24 & .87 & 0.01 \\
B $\times$ C & $\mathbf{3}$ & $\mathbf{2 , 7 0 8 . 5 5}$ & $\mathbf{7 . 3 6}$ & $<.001$ & $\mathbf{0 . 1 7}$ \\
D $\times$ C & $\mathbf{1}$ & $\mathbf{1 5 , 2 2 6 . 1 1}$ & $\mathbf{5 7 . 0 0}$ & $<.001$ & $\mathbf{0 . 6 1}$ \\
B $\times$ D $\times$ C & 3 & 602.94 & 2.54 & .06 & 0.06 \\
& $P E$ & & & & \\
Block (B) & 3 & 0.000041 & 0.03 & .99 & 0.00 \\
Distance (D) & $\mathbf{1}$ & $\mathbf{0 . 0 0 2 6 5 3}$ & $\mathbf{5 . 5 9}$ & $\mathbf{. 0 2}$ & $\mathbf{0 . 1 3}$ \\
Compatibility (C) & $\mathbf{1}$ & $\mathbf{0 . 1 4 3 7 2 8}$ & $\mathbf{2 3 . 6 5}$ & $<.001$ & $\mathbf{0 . 3 9}$ \\
B $\times$ D & $\mathbf{3}$ & $\mathbf{0 . 0 0 0 8 3 3}$ & $\mathbf{3 . 1 8}$ & $\mathbf{. 0 3}$ & $\mathbf{0 . 0 8}$ \\
B $\times$ C & 3 & 0.001739 & 2.49 & .06 & 0.06 \\
D $\times$ C & $\mathbf{1}$ & $\mathbf{0 . 0 0 6 1 2 9}$ & $\mathbf{1 7 . 2 5}$ & $<.001$ & $\mathbf{0 . 3 2}$ \\
B $\times$ D $\times$ C & $\mathbf{3}$ & $\mathbf{0 . 0 0 0 7 5 4}$ & $\mathbf{3 . 6 5}$ & $\mathbf{. 0 1}$ & $\mathbf{0 . 0 9}$ \\
\hline
\end{tabular}

Note. Boldface indicates a significant effect at alpha $=0.05$
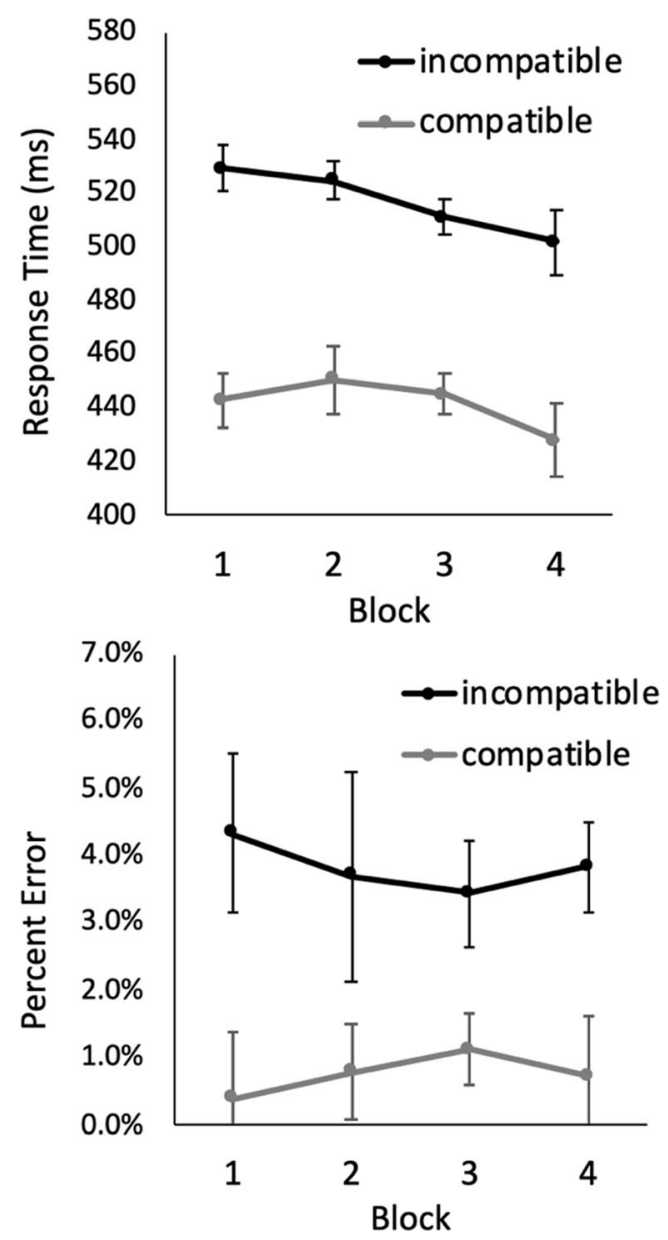

Fig. 5 Flanker effect in response time (RT) data and percentage error (PE) data from Experiment 1, graphed as a function of block (Blocks 1-3: acquisition of a consistent cue-outcome association; Block 3: reversal of the acquired association). Error bars represent $95 \%$ within-subjects confidence intervals

Distance interaction, indicating that the effect of distance increased over time (respectively, for Blocks $1-4,-0.10 \%$, $0.19 \%, 0.62 \%$, and $0.96 \%$ ). We also found an interaction between distance and compatibility, indicating larger flanker effect with near flankers (flanker effect $=3.71 \%, d_{z}=0.88$ ) compared with far flankers $\left(2.44 \%, d_{z}=0.65\right)$. We did not find a Block $\times$ Compatibility interaction. But as the trend in Fig. 5 shows, the flanker effect tended to decrease over Blocks 1-3, and it tended to increase on Block 4, consistent with our hypothesis, although this trend did not reach statistical significance. Nonetheless, the PE trend suggests that the RT results cannot be due to speed-accuracy trade-off.

\section{General discussion}

We investigated the phenomenon of sensory attenuation under different conditions using the flanker task. Participants' action (Experiments 1-3) or a visual cue (Experiment 4) was 
associated with one feature of the flankers. We found that both action-outcome contingency and cue-stimuli association can influence the effect of distractors, reflected in a reduced flanker effect with predicted flankers, during the acquisition phase, and an increased flanker effect with unpredicted flankers during the test phase.

In Experiments 1 and 2, color and distance of the flankers were associated with participants' free-choice key press, respectively. The results showed that when actions were associated with relatively simple features of the distractors (i.e., color or distance), the flankers became less distracting as the associations were acquired (Blocks 1-3) and became more distracting when the association were violated (Block 4). In Experiment 3, participants' initial key press determined target-flanker compatibility. Unlike Experiments 1 and 2, we did not find an increase of flanker effect from Block 3 to Block 4. Instead, RTs continued to decrease over the four blocks. These results indicate that action-outcome learning and its effect on distractor processing depends on the type of outcome. However, we could not, based on the null results of Experiment 3, rule out the possibility of learning with relatively complex feature, especially given that the accuracy data in Experiment 3 showed a statistically nonsignificant trend similar to the other experiments. Perhaps with a larger sample size or a more extensive learning phase, or perhaps with explicit instruction given to the participants, the same results could be observed in a variant of Experiment 3. Nonetheless, it is likely that we found a boundary condition for the sensory attenuation phenomenon. Since the predictable events in Experiment 3 could not be described as sensory events, it is rather unsurprising that an attenuation (or amplification in Block 4) was not observed. Finally, in Experiment 4, visual cues (" $\mathrm{X}$ " vs. "O") were associated with the color of flankers. We found a pattern of decrease and increase in the flanker effect similar to Experiments 1 and 2, which suggests that learning to anticipate distractor features is possible without actionoutcome learning (Press et al., 2019, 2020).

In Experiments 1-3, where a stimulus feature was associated with the free-choice response, questions regarding choice probabilities arise. Were participants more likely to choose the response that led to a distant flanker? Did they favor the response that led to target-flanker compatible trials? Assuming that participants acquired, to some degree, the responseoutcome contingencies, we should also consider whether they used those contingencies strategically. In general, it is worth investigating whether choice probabilities, causing relatively long-term predictability (e.g., flankers more frequently being green, distant, or compatible with the target), have influenced the results in an unforeseen manner. Being unable to include choice probabilities in the current analyses reflects an important weakness in the present report, and an examination of possible biases in follow-up analyses of the present data- and in future studies - choice probabilities and their influence would be worthwhile and informative.

Experiments 3-4 have an implication regarding the possibility that the learning effect might have resulted from a strategic use of the free-choice responses. Such a strategy would involve frequent selection of one choice and producing predictability of flankers in the long term (e.g., selecting one of the two free-choice response on $70 \%$ of trials would mean that the flankers become predictable, though in a way that differs from our trial-by-trial descriptions; see Logan \& Zbrodoff, 1979). Since this strategy was available in Experiment 3, where we did not observe an effect, and unavailable in Experiment 4, where we did find an effect, such a strategic use of the free-choice responses seems to be an unlikely alternative to the explanation based on associative learning. Nevertheless, this question merits a closer examination in future studies.

Our findings are consistent with various theoretical accounts of sensory attenuation (e.g., Cardoso-Leite et al., 2010; van Moorselaar \& Slagter, 2019; Waszak, CardosoLeite, \& Hughes, 2012; Yon, Gilbert, de Lange, \& Press, 2018; Yon, Zainzinger, de Lange, Eimer, \& Press, 2020). We could interpret the findings of Experiments 1-3 as an indication that acquiring action-outcome association is difficult in the case of relatively complex, relational features (perhaps requiring a more extensive learning phase) if at all possible. Alternatively, such a learning might require explicit instruction related to the contingencies, such that participants look for, and keep track of, the action-outcome associations. This possibility requires further research.

\section{Theoretical accounts}

Our findings cannot discriminate between major competing accounts of sensory attenuation, though we can still connect the present observations to more general theoretical issues. First, there is the possible link between sensory attenuation and stimulus intensity. While theoretical accounts of sensory attenuation predict reduced perceived intensity for action outcomes, their predictions depend on stimulus intensity (Roussel et al., 2013). That is because high-intensity stimuli are more likely to saturate sensory neurons, eliminating the difference made by sensory preactivation. By asking participants to discriminate stimuli with relatively low and high contrast values, Roussel et al. (2013) found larger sensory attenuation effect for low-contrast stimuli compared with high-contrast stimuli. In this regard, our findings seem ambiguous. The decrease and increase in the size of the flanker effect, as a function of learned associations, was more pronounced for far flankers (RT data in our Experiment 1, consistent with Roussel et al., 2013), though this pattern was not replicated in Experiments 2 and 4. 
A preactivation account of sensory attenuation - assuming that action selection activates the anticipated sensory outcomes of the action-predicts a selective advantage for action-congruent stimuli in terms of speed of selection, which is thought to results in phenomena such as intentional binding (Waszak et al., 2012) and faster selection of action-congruent stimuli when they are relevant to task (i.e., serve as the target of visual search; Gozli \& Ansorge, 2016; Gozli et al., 2016a; see also, Yon et al., 2018; Yon \& Press, 2017).

A recent example of how attentional selection of actioncongruent stimuli can be facilitated was reported by Nakashima (2019), who associated participants' actions with stimulus movement. Nakashima found a bias toward the location that was associated with the participants' action. Not only were participants more likely to attend to the location of their expected action-outcome, but there was also a link between the strength of bias toward the expected outcome and the participant's sense of agency (more sense of agency over action-outcome was associated with more bias toward it). It should be noted that the location of the moving stimulus in Nakashima's study was always, at least potentially, a target location. That is different from the present study, where participants were always instructed to ignore the flankers' location. Important for the theoretical accounts, the present study and Nakashima's study can both be reconciled with the preactivation account, which allows for both advantage and disadvantage of action-outcomes, depending on their task relevance (Gozli et al., 2016a).

There is also evidence that reflects the existence of two complementary processes, including both inhibition and preactivation, affecting our perception of action outcomes. Such evidence was provided by Yon and Press (2017), who asked participants to judge the brightness of the actioncongruent and action-incongruent stimuli. These stimuli were presented at different delays after actions. The authors found that the intensity of the action-congruent stimuli, compared with the action-incongruent stimuli, increased at the early stage and decreased after a delay. Similarly, Gozli and Ansorge (2016) found bias both for and against a predicted outcome, when they varied the delay between the action and the outcome. These findings suggest that the processing advantages and disadvantages of action-outcomes could be reconciled within a unified framework that includes aspects of the preactivation and the inhibition accounts (e.g., Press et al., 2019, 2020; van Moorselaar \& Slagter, 2019; Waszak et al., 2012; Yon \& Press, 2017, 2018; Yon et al., 2018; Yon et al., 2020).

\section{Relational features}

Different from our Experiments 1-2, in Experiment 3 a relation between features was associated with participants' action. The term "feature" might seem inappropriate here (Wolfe \&
Horowitz, 2017), given that participants' action determined the relationship between target and flankers, while simple features in Experiments 1 and 2 (color and distance) were independent of the target. In Experiment 3, the flanker effect was not affected by the reversal of association in Block 4. Not observing an increase in the flanker effect in Experiment 3 is evidence against action-outcome contingency learning.

According to the relational theory of attention, visual attention is guided by relative attributes, but not absolute feature value, and targets' features are not processed independently, but how they are different from the background features (Becker, 2013). According to the logic of relational features, all features can be regarded as in relation with the context: relative color difference, relative distance, and the relative orientation. Does a relational theory of perceptual features predict, in the case of Experiment 3, similar result to those of Experiments 1 and 2?

It is worth pointing out that, in Experiments 1-2, the varying flanker features were in relation to a constant reference (i.e., target color, target location), while in Experiment 3, the "reference" feature (i.e., target orientation) could itself vary on each trial. Thus, we can describe all three types of "features" as relationships, though with references that are themselves constant or changing. The target-flanker compatibility, if it is to be considered a feature at all, has to do with the relationship between target orientation and flanker orientation, whose identification occurs at a later point in time (relative to simpler features). Target orientation and flanker orientation, accordingly, cannot join and be detected, along a single feature map similar to color or distance (Treisman \& Gormican, 1988).

When features are detected against a constant background, we could think of them as "simple" features, which can preattentively guide selective attention, while complex features may not operate the preattentive visual attention (Treisman \& Gelade, 1980; Treisman \& Gormican, 1988; Wolfe, 2005). In other words, detecting simple features rely on an early-stage process that is susceptible to preactivation, attenuation (for predicted features), or amplification (for unpredicted features). This is consistent with the idea that sensory attenuation reflects early, low-level mechanisms (Roussel et al., 2014). Also relevant are the findings that the attenuated brain response started as early as 27-33 ms after stimulus onset, which reveal early low-level auditory processing of predicted stimuli and confirm early-stage operation of the forward model and the efference copy (Bäß et al., 2009; Wolpert \& Flanagan, 2001).

\section{Action-outcome learning versus associative learning}

In our control Experiment 4, we found a similar pattern of results to Experiments 1-2 - namely, decreased flanker effect over Blocks 1-3 and an increase when the contingencies were 
reversed in the last block. These results suggest that perceptual associative learning can induce a similar bias that arises from action-outcome learning. This seems inconsistent with previous finding that attentional bias to action-related stimuli is different from expectation in general, and is linked to a sense of agency over predicted stimuli (Nakashima, 2019). The findings are also inconsistent with the findings of Weiss and Schütz-Bosbach (2012), who designed an experiment with three conditions: (1) self-generated action condition, (2) other-generated-unanticipated condition, and (3) othergenerated-anticipated condition. They found that attenuation only in self-generated action condition. Moreover, CardosoLeite et al. (2010) conducted an experiment similar to the present control experiment. Rather than associating two visual stimuli, as in the present study, they associated Gabor orientation with high-pitch and low-pitch tones. They found no evidence of learned association between tones and Gabor stimuli, which they interpreted as a disadvantage for perceptual learning compared with action-outcome learning.

\section{Conclusion}

The present study shows that learning action-outcome contingencies can change distractor processing, though this learning does not apply equally to all types of outcome. While actionoutcome contingencies with relatively simple features (the color \& distance of the flankers) resulted in modulation of the flanker effect (indicating learned associations), the same was not observed with a relatively complex, relational feature (target-flanker compatibility). Finally, we found a similar pattern of associative learning with cue-outcome contingencies, which suggests, at least in the case of the present task, actions might not have a special status in associative learning.

\section{References}

Aliu, S. O., Houde, J. F., \& Nagarajan, S. S. (2009). Motor-induced suppression of the auditory cortex. Journal of Cognitive Neuroscience, 21, 791-802.

Bäß, P., Jacobsen, T., \& Schröger, E. (2008). Suppression of the auditory N1 event-related potential component with unpredictable selfinitiated tones: Evidence for internal forward models with dynamic stimulation. International Journal of Psychophysiology, 70(2), 137143.

Bäß, P., Widmann, A., Roye, A., Schröger, E., \& Jacobsen, T. (2009). Attenuated human auditory middle latency response and evoked 40$\mathrm{Hz}$ response to self-initiated sounds. European Journal of Neuroscience, 29(7), 1514-1521.

Becker, S. I. (2013). Why you cannot map attention: A relational theory of attention and eye movements. Australian Psychologist, 48(6), 389-398.

Becker, S. I., Folk, C. L., \& Remington, R. W. (2013). Attentional capture does not depend on feature similarity, but on target-nontarget relations. Psychological Science, 24(5), 634-647.
Blakemore, S. J., Wolpert, D., \& Frith, C. (2000). Why can't you tickle yourself? NeuroReport, 11(11), R11-R16.

Blakemore, S. J., Wolpert, D. M., \& Frith, C. D. (1998). Central cancellation of self-produced tickle sensation. Nature Neuroscience, 1(7), 635-640.

Bompas, A., \& O'Regan, J. K. (2006a). Evidence for a role of action in colour perception. Perception, 35(1), 65-78.

Bompas, A., \& O'Regan, J. K. (2006b). More evidence for sensorimotor adaptation in color perception. Journal of Vision, 6(2), 145-153.

Cardoso-Leite, P., Mamassian, P., Schütz-Bosbach, S., \& Waszak, F. (2010). A new look at sensory attenuation: Action-effect anticipation affects sensitivity, not response bias. Psychological Science, 21(12), 1740-1745.

Clark, A. (2013). Whatever next? Predictive brains, situated agents, and the future of cognitive science. Behavioral and Brain Sciences, $36(3), 181-204$.

Cousineau, D. (2005). Confidence intervals in within-subject designs: A simpler solution to Loftus and Masson's method. Tutorials in Quantitative Methods for Psychology, 1(1), 42-45.

Dignath, D., Kiesel, A., Frings, C., \& Pastötter, B. (2019). Electrophysiological evidence for action-effect prediction. Journal of Experimental Psychology: General. Advance online publication. https://doi.org/10.1037/xge0000707

Eriksen, B. A., \& Eriksen, C. W. (1974). Effects of noise letters upon the identification of a target letter in a nonsearch task. Perception \& Psychophysics, 16(1), 143-149.

Ford, J. M., Gray, M., Faustman, W. O., Heinks, T. H. \& Mathalon, D. H. (2005). Reduced gamma-band coherence to distorted feedback during speech when what you say is not what you hear. International Journal of Psychophysiology, 57, 143-150.

Ford, J. M., Mathalon, D. H., Heinks, T., Kalba, S., Faustman, W. O., \& Roth, W. T. (2001a). Neurophysiological evidence of corollary discharge dysfunction in schizophrenia. American Journal of Psychiatry, 158, 2069-2071.

Ford, J. M., Mathalon, D. H., Kalba, S., Whitfield, S., Faustman, W. O., \& Roth, W. T. (2001b). Cortical responsiveness during talking and listening in schizophrenia: An event-related brain potential study. Biological Psychiatry, 50, 540-549.

Gozli, D. (2019). Free Choice. In Experimental psychology and human agency (pp. 113-136). Cham: Springer.

Gozli, D. G., \& Ansorge, U. (2016). Action selection as a guide for visual attention. Visual Cognition, 24(1), 38-50.

Gozli, D. G., Aslam, H., \& Pratt, J. (2016a). Visuospatial cueing by selfcaused features: Orienting of attention and action-outcome associative learning. Psychonomic Bulletin \& Review, 23(2), 459--467.

Gozli, D. G., Huffman, G., \& Pratt, J. (2016b). Acting and anticipating: Impact of outcome-compatible distractor depends on response selection efficiency. Journal of Experimental Psychology: Human Perception \& Performance, 42(10), 1601-1614.

Heinks-Maldonado, T. H., Mathalon, D. H., Gray, M., \& Ford, J. M. (2005). Fine-tuning of auditory cortex during speech production. Psychophysiology, 42, 180-190.

Heinks-Maldonado, T. H., Nagarajan, S. S., \& Houde, J. F. (2006) Magnetoencephalographic evidence for a precise forward model in speech production. NeuroReport, 17, 1375-1379.

Hommel, B., Müsseler, J., Aschersleben, G., \& Prinz, W. (2001). The theory of event coding (TEC): A framework for perception and action planning. Behavioral and Brain Sciences, 24(5), 849-878.

Houde, J. F., Nagarajan, S. S., Sekihara, K., \& Merzenich, M. M. (2002). Modulation of the auditory cortex during speech: An MEG study. Journal of Cognitive Neuroscience, 14(8), 1125-1138.

Huffman, G., Gozli, D. G., Hommel, B., \& Pratt, J. (2019). Response preparation, response selection difficulty, and response-outcome learning. Psychological Research, 83(2), 247-257. 
Hughes, G., \& Waszak, F. (2011). ERP correlates of action effect prediction and visual sensory attenuation in voluntary action. NeuroImage, 56(3), 1632-1640

Itti, L., \& Baldi, P. (2009). Bayesian surprise attracts human attention. Vision Research, 49(10), 1295-1306.

James, W. (1890). The principles of psychology (Vols. 1 \& 2). New York, NY: Henry Holt \& Co.

Kramer, A. F., \& Jacobson, A. (1991). Perceptual organization and focused attention: The role of objects and proximity in visual processing. Perception \& Psychophysics, 50(3), 267-284.

Logan, G. D., \& Zbrodoff, N. J. (1979). When it helps to be misled: Facilitative effects of increasing the frequency of conflicting stimuli in a Stroop-like task. Memory \& Cognition, 7(3), 166-174.

Logan, G. D., \& Zbrodoff, N. J. (1982). Constraints on strategy construction in a speeded discrimination task. Journal of Experimental Psychology: Human Perception and Performance, 8(4), 502-520.

Lotze, R. H. (1852). Medical psychology, or, physiology of the soul. Weidmann, Leipzig, 4.

Melcher, T., Weidema, M., Eenshuistra, R. M., Hommel, B., \& Gruber, O. (2008). The neural substrate of the ideomotor principle: An event-related fMRI analysis. NeuroImage, 39(3), 1274-1288.

Melcher, T., Winter, D., Hommel, B., Pfister, R., Dechent, P., \& Gruber, O. (2013). The neural substrate of the ideomotor principle revisited: Evidence for asymmetries in action-effect learning. Neuroscience, $231,13-27$.

Miall, R. C., \& Wolpert, D. M. (1996). Forward models for physiological motor control. Neural Networks, 9(8), 1265-1279.

Morey, R. D. (2008). Confidence intervals from normalized data: A correction to Cousineau (2005). Tutorials in Quantitative Methods for Psychology, 4(2), 61-64.

Naefgen, C., \& Janczyk, M. (2018). Free choice tasks as random generation tasks: An investigation through working memory manipulations. Experimental Brain Research, 236(8), 2263-2275.

Nakashima, R. (2019). Beyond one's body parts: Remote object movement with sense of agency involuntarily biases spatial attention. Psychonomic Bulletin \& Review, 26(2), 576-582.

Nieuwenhuis, S., Stins, J. F., Posthuma, D., Polderman, T. J., Boomsma, D. I., \& de Geus, E. J. (2006). Accounting for sequential trial effects in the flanker task: Conflict adaptation or associative priming? Memory \& Cognition, 34(6), 1260-1272.

Numminen, J., \& Curio, G. (1999). Differential effects of overt, covert and replayed speech on vowel-evoked responses of the human auditory cortex. Neuroscience Letters, 272, 29-32.

Numminen, J., Salmelin, R., \& Hari, R. (1999). Subject's own speech reduces reactivity of the human auditory cortex. Neuroscience Letters, 265, 119-122.

Pearce, J. M., \& Hall, G. (1980). A model for Pavlovian learning: variations in the effectiveness of conditioned but not of unconditioned stimuli. Psychological Review, 87(6), 532.

Pfister, R., Kiesel, A., \& Melcher, T. (2010). Adaptive control of ideomotor effect anticipations. Acta Psychologica, 135(3), 316-322.

Pfister, R., Kiesel, A., \& Hoffmann, J. (2011). Learning at any rate: Action-effect learning for stimulus-based actions. Psychological Research, 75(1), 61-65.

Pfister, R., Heinemann, A., Kiesel, A., Thomaschke, R., \& Janczyk, M. (2012). Do endogenous and exogenous action control compete for perception? Journal of Experimental Psychology: Human Perception and Performance, 38(2), 279-284.

Press, C., Kok, P., \& Yon, D. (2019). The perceptual prediction paradox. Trends in Cognitive Sciences, 24(1), 13-24.

Press, C., Kok, P., \& Yon, D. (2020). Learning to perceive and perceiving to learn. Trends in Cognitive Sciences, 24(4), 260-261.

Rao, R. P., \& Ballard, D. H. (1999). Predictive coding in the visual cortex: A functional interpretation of some extra-classical receptive-field effects. Nature Neuroscience, 2(1), 79-87.
Roussel, C., Hughes, G., \& Waszak, F. (2013). A preactivation account of sensory attenuation. Neuropsychologia, 51(5), 922-929.

Roussel, C., Hughes, G., \& Waszak, F. (2014). Action prediction modulates both neurophysiological and psychophysical indices of sensory attenuation. Frontiers in Human Neuroscience, 8, 115.

Schafer, E. W., \& Marcus, M. M. (1973). Self-stimulation alters human sensory brain responses. Science, 181(4095), 175-177.

Schmidts, C., Foerster, A., \& Kunde, W. (2018). Conflict modification: Predictable production of congruent situations facilitates responding in a Stroop task. Psychological Research, 83, 1722-1732.

Schneider, D. W. (2018). Alertness and cognitive control: Testing the early onset hypothesis. Journal of Experimental Psychology: Human Perception \& Performance, 44(5), 756-766.

Treisman, A., \& Gelade, G. (1980). A feature-integration theory of attention. Cognitive Psychology, 12, 97-136. https://doi.org/10.1016/ 0010-0285(80)90005-5

Treisman, A., \& Gormican, S. (1988). Feature analysis in early vision: Evidence from search asymmetries. Psychological Review, 95(1), $15-48$.

van Moorselaar, D., \& Slagter, H. A. (2019). Learning what is irrelevant or relevant: Expectations facilitate distractor inhibition and target facilitation through distinct neural mechanisms. Journal of Neuroscience, 39(35), 6953-6967.

Waszak, F., Cardoso-Leite, P., \& Hughes, G. (2012). Action effect anticipation: Neurophysiological basis and functional consequences. Neuroscience \& Biobehavioral Reviews, 36(2), 943-959.

Weiss, C., \& Schütz-Bosbach, S. (2012). Vicarious action preparation does not result in sensory attenuation of auditory action effects. Consciousness \& Cognition, 21(4), 1654-1661.

Wolfe, J. M. (2005). Guidance of visual search by preattentive information. In J. Tsotsos, G. Rees, \& L. Itti (Eds.), Neurobiology of attention (pp. 101-104). Cambridge, MA: Academic Press.

Wolfe, J. M., \& Horowitz, T. S. (2017). Five factors that guide attention in visual search. Nature Human Behaviour, 1(3), 1-8.

Wolfensteller, U., \& Ruge, H. (2014). Response selection difficulty modulates the behavioral impact of rapidly learnt action effects. Frontiers in Psychology, 5, 1382.

Wolpert, D. M., \& Flanagan, J. R. (2001). Motor prediction. Current Biology, 11(18), R729-R732.

Wolpert, D. M., \& Kawato, M. (1998). Multiple paired forward and inverse models for motor control. Neural Networks, 11(7/8), $1317-1329$.

Wolpert, D. M., Ghahramani, Z., \& Jordan, M. I. (1995). An internal model for sensorimotor integration. Science, 269(5232), 1880 1882.

Wykowska, A., Schubö, A., \& Hommel, B. (2009). How you move is what you see: Action planning biases selection in visual search. Journal of Experimental Psychology: Human Perception \& Performance, 35(6), 1755.

Yon, D., Gilbert, S. J., de Lange, F. P., \& Press, C. (2018). Action sharpens sensory representations of expected outcomes. Nature Communications, 9(1), 1-8.

Yon, D., \& Press, C. (2017). Predicted action consequences are perceptually facilitated before cancellation. Journal of Experimental Psychology: Human Perception \& Performance, 43(6), 1073-83.

Yon, D., \& Press, C. (2018). Sensory predictions during action support perception of imitative reactions across suprasecond delays. Cognition, 173, 21-27.

Yon, D., Zainzinger, V., de Lange, F. P., Eimer, M., \& Press, C. (2020). Action biases perceptual decisions toward expected outcomes. Journal of Experimental Psychology: General. https://doi.org/10. 31234/osf.io/3zp8n

Publisher's note Springer Nature remains neutral with regard to jurisdictional claims in published maps and institutional affiliations. 